\title{
Stage 4S Neuroblastoma: What Are the Outcomes? A Systematic Review of Published Studies
}

\author{
Arimatias Raitio ${ }^{1,2}$ Michael J. Rice ${ }^{1}$ Dhanya Mullassery ${ }^{1}$ Paul D. Losty ${ }^{1,3}$ \\ ${ }^{1}$ Department of Paediatric Surgery, Alder Hey Children's NHS \\ Foundation Trust, Liverpool, Merseyside, United Kingdom \\ 2 Department of Paediatric Surgery, University of Turku, Turku, \\ Finland \\ ${ }^{3}$ Institute of Translational Medicine, University of Liverpool, \\ Liverpool, Merseyside, United Kingdom \\ Eur J Pediatr Surg 2021;31:385-389. \\ Address for correspondence Paul D. Losty, MD, FRCS, FEBPS, \\ Department of Paediatric Surgery, Alder Hey Children's NHS \\ Foundation Trust, Liverpool L12 2AP, United Kingdom \\ (e-mail: paul.losty@liverpool.ac.uk).
}

\begin{abstract}
Keywords

- neuroblastoma

- INRG stage MS

- outcome

- stage $4 \mathrm{~S}$

Introduction The prognosis of stage 4S/MS neuroblastoma has traditionally been reported as excellent, yet conflicting treatment protocols exist for this enigmatic disease. To critically address this question, we have undertaken a systematic review of published studies to accurately determine outcomes for infants with stage 4S/MS neuroblastoma.

Materials and Methods Studies were identified using MEDLINE, Embase, and Cochrane databases using the relevant search terms. Literature reviews, case reports, and adult studies were excluded. Data were extracted independently following article selection by three authors and reviewed by the senior author.

Results The original search retrieved 2,325 articles. Following application of exclusion criteria and removing duplicate data, 37 studies ( 1,105 patients) were included for final review. Overall patient survival was $84 \%$. Twelve studies ( 544 patients) recorded MYCN status. Mortality in MYCN amplified tumors was $56 \%$. Chromosome $1 \mathrm{p} / 11 \mathrm{q}$ status was reported in four studies and $1 \mathrm{p} / 11 \mathrm{q}$ deletion carried a $40 \%$ fatality rate. Management included observation only (201 patients, $8.5 \%$ mortality), surgical resection of primary tumor only ( 153 patients, $6.5 \%$ mortality), chemotherapy only (186 patients, $21 \%$ mortality), radiotherapy ( 5 deaths, 33\% mortality), chemotherapy with surgery (160 patients, $10 \%$ mortality), surgery with radiotherapy ( 21 patients, $19 \%$ mortality), radiotherapy with chemotherapy (42 patients, $29 \%$ mortality), and surgery with chemotherapy and radiotherapy (27 patients, 33\% mortality).

Conclusion There is a significant mortality observed in stage 4S/MS neuroblastoma infants with a dismal outcome observed in those patients with MYCN amplification and $1 \mathrm{p} / 11 \mathrm{q}$ deletion. Those patients suitably amenable for conservative management or surgery to excise the primary tumor carry the best prognosis.
\end{abstract}

received

June 29, 2020

accepted

August 16, 2020

published online

September 15, 2020 


\section{Introduction}

Stage 4S neuroblastoma was first described in 1971 by D'Angio et al and referred to very young patients with otherwise stage I or II disease, but with metastasis in the liver, skin, or bone marrow. ${ }^{1}$ The International Neuroblastoma Risk Group (INRG) staging system has also reclassified 4S as stage MS and sets a patient age upper limit here at 18 months. ${ }^{2,3}$ Stage $4 \mathrm{~S}$ neuroblastoma disease (MS stage) is typically characterized by an initial phase of rapid tumor progression followed by spontaneous regression in most cases. ${ }^{4}$ However, disease progression regardless of any therapy(s) deployed may be seen in only a minority of patients and survival rates reported in the literature varyingly range from 56 to $90 \%$ cases. $^{5-9}$

There is evidence that very young age at diagnosis $(<2$ months), ${ }^{10,11}$ life-threatening symptoms, ${ }^{12}$ MYCN amplification, ${ }^{11,13}$ and chromosome $1 \mathrm{p}$ deletions ${ }^{13}$ are predictors of poor outcome in stage $4 \mathrm{~S}$ neuroblastoma (stage MS). However, optimal treatment strategies and their outcomes still remain poorly understood. ${ }^{8}$

Against this background, the aim of this systematic review study was to accurately better define clinical outcomes of infants with stage $4 \mathrm{~S}$ (stage MS) neuroblastoma taking into account the different treatment modalities employed with the biological features of this enigmatic neuroblastic tumor.

\section{Materials and Methods}

\section{Identification and Selection of Studies}

A comprehensive search of the published literature in MEDLINE, Embase, and Cochrane database(s) was performed based on Preferred Reporting Items for Systematic Reviews and Meta-Analyses guidelines. ${ }^{14}$ Search was made using term "neuroblastoma" in combination with one of the following keywords: " $4 S$ " or "IVS" or "stage $4 S$ " or "MS" or "stage IVS" or "infant" or "neonate" or "spontaneous regression" or "congenital." All articles published up to May 15, 2020, were included in the review.

\section{Inclusion and Exclusion Criteria}

This study included all original articles reporting on outcomes of stage 4S (INRG stage MS) neuroblastoma. NonEnglish articles and case reports ( $<3$ patients) were first excluded with title and abstract screening. Studies with no stage $4 \mathrm{~S}$ patients and/or survival data were also excluded (-Fig. 1).

\section{Data Extraction and Analysis}

Identified articles were independently reviewed by three authors, and final selection was approved by the senior author. The data on the survival of patients with stage $4 \mathrm{~S}$ (INRG stage MS) neuroblastoma were then extracted from the original publications. Data on the treatment modalities of stage 4S (INRG stage MS) neuroblastoma, MYCN, and chromosome $1 \mathrm{p} / 11 \mathrm{q}$ deletion status were also included in the analyses where available.

\section{Statistical Analysis}

Chi-square and Fisher's exact tests were utilized to analyze categorical variables. A significance level of $p \leq 0.05$ (twotailed) was set. Analyses were performed using JMP Pro, version 13.1.0 for Windows (SAS Institute Inc., Cary, North Carolina, United States).

\section{Results}

The original search through different databases retrieved 2,325 articles. A total of 1,623 studies were evaluated in screening of titles and abstracts after duplicates were excluded. Eighty-five articles met the inclusion criteria in screening and were selected for full-text review. After fulltext review of 85 articles, 37 articles met the eligibility criteria and were selected for review ( - Fig. 1 ). The published studies covered the time period(s) from 1971 to 2020.

In total, there were 1,105 patients with stage 4 S (INRG stage MS) neuroblastoma identified with overall survival of $84 \%$. The most common site of primary tumor location was the adrenal gland with metastasis observed in the liver. MYCN status was fully reported in 12 studies including 544 patients and MYCN amplification here carried 56\% mortality. Chromosome $1 \mathrm{p} / 11 \mathrm{q}$ deletions were only reported in three studies and 133 patients with $1 \mathrm{p} / 11 \mathrm{q}$ deletion carried a $40 \%$ fatality rate ( - Table 1 ).

A total of 201 patients were managed by observation only with an $8.5 \%$ mortality. Surgical resection of the primary tumor was performed on 153 patients with $6.5 \%$ fatality rate and surgery with chemotherapy on 160 patients with $10 \%$ mortality. The above-mentioned three treatment groups had significantly better outcome(s) compared with other treatment modalities listed $(p<0.001)$ ( - Table 2$)$. One hundred eighty-six patients were treated with chemotherapy only with $21 \%$ mortality.

\section{Discussion}

This systematic review demonstrates that stage 4S (INRG stage MS) neuroblastoma carries the best prognosis in only those groups of patients amenable for observation only or surgical resection of primary tumor with or without chemotherapy. Moreover, MYCN amplification and chromosome $1 \mathrm{p} / 11 \mathrm{q}$ deletion were both predictors of mortality.

Observation only was the most commonly used treatment for stage $4 \mathrm{~S}$ neuroblastoma. Spontaneous regression or differentiation to a ganglioneuroma phenotype is common in stage 4S (INRG stage MS) neuroblastoma with "benign" molecular biology. ${ }^{4}$ Here, most tumors can be treated with active observation only with modest outcome(s) anticipated including stage 4 S (INRG stage MS) neuroblastoma mortality ranging from 0 to $19 \%^{8,15-18}$

Surgery with or without chemotherapy yielded excellent outcome(s) in stage 4S (INRG stage MS) neuroblastoma according to the quality of published literature reviewed here in this systematic review. Patients suitable for surgical resection of the offending primary tumor only had the best 

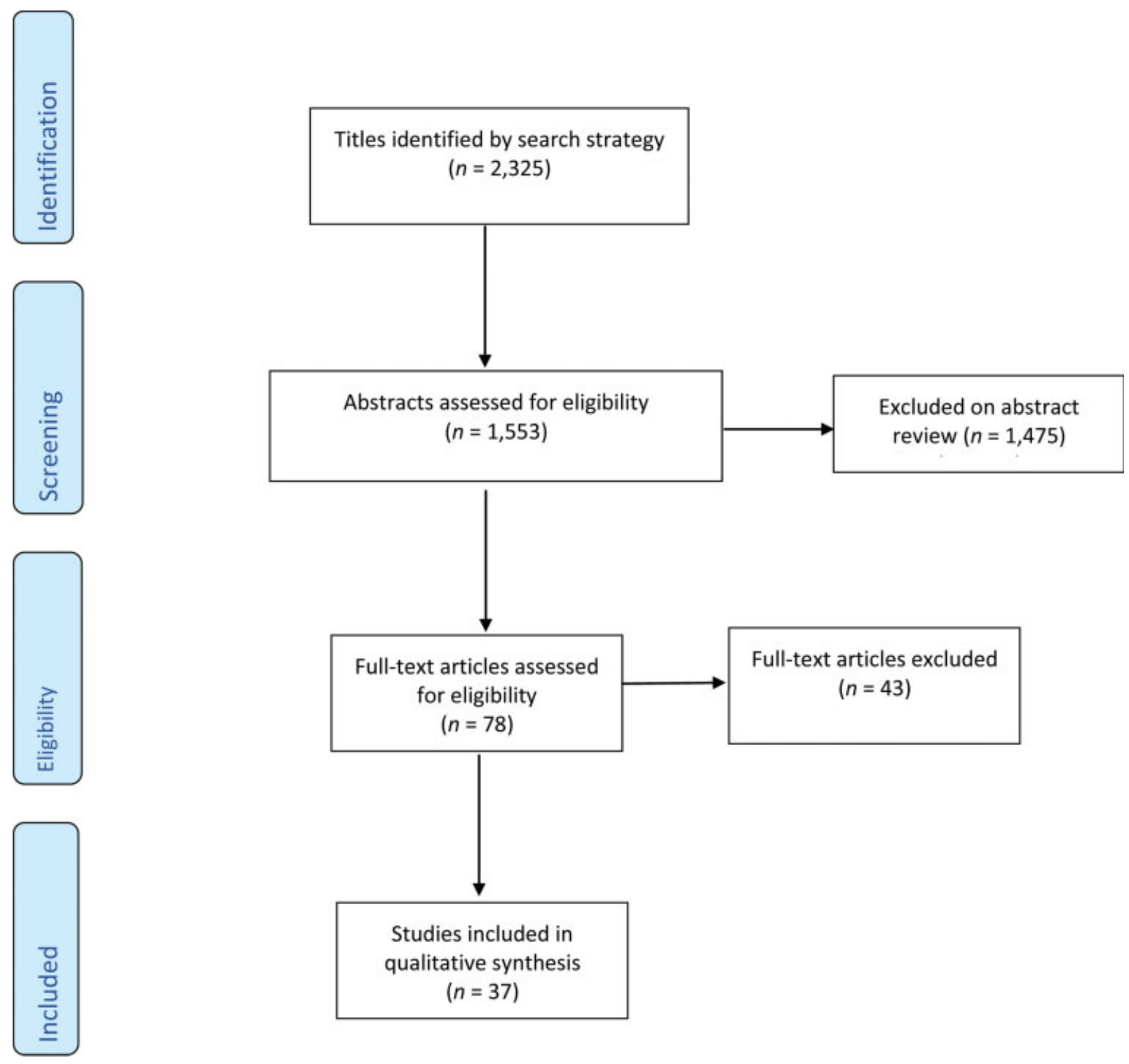

Fig. 1 Preferred Reporting Items for Systematic Reviews and Meta-Analyses study selection flow diagram.

outcome(s). ${ }^{8,11,15,17}$ Those requiring neoadjuvant chemotherapy before definitive surgical resection had also good outcomes with only $10 \%$ mortality. ${ }^{11,15,19,20}$ Interestingly, those treated with surgical resection and radiotherapy with or without chemotherapy likely "scaled up" to control fulminant liver metastases had significantly worse outcome(s).

Patients with stage 4S (INRG stage MS) neuroblastoma treated with chemotherapy only had significant mortality compared with those treated with observation or surgery.
Chemotherapy only was the second most common treatment identified in this systematic review and reported mortality varied significantly ranging from 0 to $29 \% .8,11,17,21$ We postulate that the inferior outcome(s) associated with chemotherapy are most likely reflective of the unfavorable anatomical site of tumor and/or their unique molecular tumor characteristics. $^{22}$

Radiotherapy alone was administered in 15 patients with $33 \%$ mortality (range: $0-100 \%)^{12,17,19,23}$ This review of

Table 1 Association of molecular biology and survival in 4S neuroblastoma

\begin{tabular}{|l|l|l|l|l|}
\hline & Number of cases $(\boldsymbol{n})$ & Deaths $(\boldsymbol{n})$ & Mortality (\%) & $\boldsymbol{p}$-Value \\
\hline MYCN amplification & 36 & 20 & 55.6 & $<0.001$ \\
\hline MYCN not amplified & 498 & 53 & 10.6 & 0.02 \\
\hline Chromosome 1p/11q deletion & 10 & 4 & 40.0 & 8.9 \\
\hline Normal chromosome 1p/11q & 123 & 11 & 8.9 \\
\hline
\end{tabular}


Table 2 Survival of stage 4 S neuroblastoma with different treatment modalities

\begin{tabular}{|l|l|l|l|}
\hline & Number of cases $(\boldsymbol{n})$ & Deaths $(\boldsymbol{n})$ & Mortality (\%) \\
\hline Observation & 201 & 17 & 8.5 \\
\hline Surgery only & 153 & 10 & 6.5 \\
\hline Surgery and chemotherapy & 160 & 16 & 10.0 \\
\hline Chemotherapy only & 186 & 39 & 21.0 \\
\hline Radiotherapy only & 15 & 5 & 33.3 \\
\hline Surgery and radiotherapy & 21 & 4 & 19.0 \\
\hline Radiotherapy and chemotherapy & 42 & 12 & 28.6 \\
\hline Surgery, chemotherapy, and radiotherapy & 27 & 9 & 33.3 \\
\hline Overall & 1,105 & 174 & 15.7 \\
\hline
\end{tabular}

published studies therefore shows that radiotherapy alone is associated with poor prognosis. Similarly, combination(s) of chemotherapy and radiotherapy were likewise associated with a significant fatality rate of $29 \%$. $111,12,17,23$

A lack of robust published data showing "evidence-based" selection criteria of deployed therapy strategies for patients with stage 4S (INRG stage MS) neuroblastoma is a main limitation of this current study. Fully comparing outcomes metrics of the varying molecular characteristics of the stage 4 S (INRG stage MS) tumors between treatment groups were challenging due to limited information available. Finally, all included studies analyzed were retrospective cohort populations.

\section{Conclusion}

In conclusion, this study therefore demonstrates that stage 4S (INRG stage MS) neuroblastoma is associated with good outcome(s) in most cases. Molecular characteristics of the $4 \mathrm{~S}$ (INRG stage MS) neuroblastic tumor are the best predictors of mortality. Those patients amenable for observation or surgical resection of primary tumor appear to have the best overall prognosis.

\section{Conflict of Interest}

Dr. Raitio reports grants from Emil Aaltonen Foundation and Turku University Foundation, outside the submitted work.

\section{References}

1 D'Angio GJ, Evans AE, Koop CE. Special pattern of widespread neuroblastoma with a favourable prognosis. Lancet 1971;1 (7708):1046-1049

2 Cohn SL, Pearson AD, London WBINRG Task Force, et al; The International Neuroblastoma Risk Group (INRG) classification system: an INRG Task Force report. J Clin Oncol 2009;27(02):289-297

3 Monclair T, Brodeur GM, Ambros PFINRG Task Force, et al; The International Neuroblastoma Risk Group (INRG) staging system: an INRG Task Force report. J Clin Oncol 2009;27(02):298-303

4 Brodeur GM. Spontaneous regression of neuroblastoma. Cell Tissue Res 2018;372(02):277-286

5 Koivusalo AI, Pakarinen MP, Rintala RJ, Saarinen-Pihkala UM. Surgical treatment of neuroblastoma: twenty-three years of experience at a single institution. Surg Today 2014;44(03): 517-525

6 Moreno F, Lopez Marti J, Palladino M, Lobos P, Gualtieri A, Cacciavillano W. Childhood neuroblastoma: incidence and survival in Argentina. Report from the National Pediatric Cancer Registry, ROHA Network 2000-2012. Pediatr Blood Cancer 2016; 63(08):1362-1367

7 Youlden DR, Frazier AL, Gupta S, et al. Stage at diagnosis for childhood solid cancers in Australia: a population-based study. Cancer Epidemiol 2019;59:208-214

8 De Bernardi B, Di Cataldo A, Garaventa A, et al. Stage 4 s neuroblastoma: features, management and outcome of 268 cases from the Italian Neuroblastoma Registry. Ital J Pediatr 2019;45(01):8

9 Salim A, Mullassery D, Pizer B, McDowell HP, Losty PD. Neuroblastoma: a 20-year experience in a UK regional centre. Pediatr Blood Cancer 2011;57(07):1254-1260

10 De Bernardi B, Pianca C, Boni LItalian Cooperative Group on Neuroblastoma, et al; Disseminated neuroblastoma (stage IV and IV-S) in the first year of life. Outcome related to age and stage. Cancer 1992;70(06):1625-1633

11 Katzenstein HM, Bowman LC, Brodeur GM, et al. Prognostic significance of age, MYCN oncogene amplification, tumor cell ploidy, and histology in 110 infants with stage $\mathrm{D}(\mathrm{S})$ neuroblastoma: the pediatric oncology group experience-a pediatric oncology group study. J Clin Oncol 1998;16(06):2007-2017

12 Hsu LL, Evans AE, D’Angio GJ. Hepatomegaly in neuroblastoma stage 4s: criteria for treatment of the vulnerable neonate. Med Pediatr Oncol 1996;27(06):521-528

13 Schleiermacher G, Rubie H, Hartmann ONeuroblastoma Study Group of the French Society of Paediatric Oncology, et al; Treatment of stage $4 \mathrm{~s}$ neuroblastoma-report of 10 years' experience of the French Society of Paediatric Oncology (SFOP). Br J Cancer 2003;89(03):470-476

14 Moher D, Liberati A, Tetzlaff J, Altman DGPRISMA Group. Preferred Reporting Items for Systematic Reviews and Meta-Analyses: the PRISMA statement. BMJ 2009;339:b2535

15 De Bernardi B, Gerrard M, Boni L, et al. Excellent outcome with reduced treatment for infants with disseminated neuroblastoma without MYCN gene amplification. J Clin Oncol 2009;27(07): 1034-1040

16 Fischer M, Oberthuer A, Brors B, et al. Differential expression of neuronal genes defines subtypes of disseminated neuroblastoma with favorable and unfavorable outcome. Clin Cancer Res 2006;12 (17):5118-5128

17 Nickerson HJ, Matthay KK, Seeger RC, et al. Favorable biology and outcome of stage IV-S neuroblastoma with supportive care or minimal therapy: a Children's Cancer Group study. J Clin Oncol 2000;18(03):477-486 
18 Fawzy M, El Zomor H, El Menawi S, et al. Watch and see strategy in selected neuroblastoma case scenarios: success and limitations. J Pediatr Hematol Oncol 2019;41(06):e384-e387

19 de Bouyn-Icher C, Minard-Colin V, Isapof A, Khuong Quang DA, Redon I, Hartmann O. Malignant solid tumors in neonates: a study of 71 cases [in French]. Arch Pediatr 2006;13(12):1486-1494

20 Wang Z, Sun $\mathrm{H}$, Li K, et al. Prognostic factor analysis of stage $4 \mathrm{~S}$ neuroblastoma in infant patients: a single center study. J Pediatr Surg 2019;54(12):2585-2588
21 Weintraub M, Waldman E, Koplewitz B, et al. A sequential treatment algorithm for infants with stage $4 \mathrm{~s}$ neuroblastoma and massive hepatomegaly. Pediatr Blood Cancer 2012;59(01): 182-184

22 Salim A, Raitio A, Mullassery D, Pizer B, Losty PD. Neuroblastoma: The Association of Anatomical Tumour Site, Molecular Biology and Patient Outcomes. Eur J Ped Surg 2020 (submitted)

23 Stokes SH, Thomas PR, Perez CA, Vietti TJ. Stage IV-S neuroblastoma. Results with definitive therapy. Cancer 1984;53(10):2083-2086 